\title{
SUR UNE QUESTION DE LOCALISATION
}

\author{
par M. P. MALLIAVIN-BRAMERET
}

(Received 7 October, 1979)

Il est connu [4] que si $A=U(g)$ est l'algèbre enveloppante d'une algèbre de Lie nilpotente $\mathfrak{g}$ de dimension finie sur un corps $F$ de caractéristique 0 , tout idéal (complètement) premier $P$ a pour localisé $R=A_{\mathrm{p}}$ un anneau régulier au sens de [5]; c'està-dire que le radical de Jacobson $\mathfrak{M}$ de $R$ est engendré par une suite centralisante régulière de longueur $n=\mathrm{K}$-dim $R$, soit $\left(z_{1}, \ldots, z_{n}\right)$. Dans le cas très particulier où $P$ est l'idéal d'augmentation de $U(\mathrm{~g})$ il suffit de prendre pour $\left(z_{1}, \ldots, z_{n}\right)$ l'image dans $U(\mathrm{~g})_{P}$ d'une base de $\mathrm{g}$ sur $F$ adaptée à la suite centrale ascendante de $\mathrm{g}$.

Un problème qu'il est alors naturel de se poser est de savoir si étant donné $A, P, R$, $\mathfrak{W}=\left(z_{1}, \ldots, z_{n}\right)$ comme précédemment et étant donné $1 \leqslant t \leqslant n$, l'anneau $R /\left(z_{1}, \ldots, z_{t}\right)$ localisé en un idéal premier quelconque est régulier au sens précédent.

En adoptant le raisonnement de [4], T. Levasseur a donné pour $t=1$ une réponse positive.

Nous nous proposons de donner une réponse positive au problème précédent, pour tout $t$ dans le cas où $P$ est un idéal maximal et où le corps $F$ est algébriquement clos.

Dans toute la suite les anneaux considérés sont unitaires, noethériens à droite et à gauche, leurs idéaux premiers sont complètement premiers et localisables au sens classique.

DÉFINITION. Soit $R$ (resp. $U(g)$ ) un anneau local régulier au sens de Walker (respectivement une algèbre enveloppante d'une algèbre de Lie nilpotente $g$ de dimension finie sur un corps $F$ ) $\mathfrak{R}$ le radical de $R$ (respectivement $P$ un idéal maximal de $U(\mathfrak{g})$ ). Un système régulier de générateurs de $\mathfrak{M}$ (respectivement $P$ ) soit $z_{1}, \ldots, z_{n}$, est dit privilégié si pour tout $t, 1 \leqslant t \leqslant n$ l'anneau quotient $R /\left(z_{1}, \ldots, z_{t}\right)$ (respectivement $U(\mathrm{~g}) /\left(z_{1}, \ldots, z_{t}\right)$ ) localisé en un idéal premier quelconque est régulier.

Evidemment si l'idéal maximal $P$ de $U(\mathrm{~g})$ possède un système privilégié de générateurs, alors le radical de l'anneau $R=U(\mathrm{~g})_{P}$ en possède un aussi.

On va démontrer que l'idéal maximal $P$ de $U(\mathfrak{g})$ possède un système privilégié de générateurs, lorsque le corps de base $F$ est algébriquement clos.

Il est connu [3] que $P$ possède un système centralisant de générateurs. On utilisera ici une démontration calquée sur celle de [3]. On commencera par rappeler la proposition suivante [2].

Proposrrion 1 (T. Levasseur). Soit g une algèbre de Lie nilpotente sur un corps $F$ de caractéristique 0 (non nécessairement algébriquement clos). Soit $Q$ un idéal premier de l'algèbre enveloppante $U(\mathrm{~g})$, soit $\left(z_{1}, \ldots, z_{t}\right)$ un système centralisant et régulier de générateurs du radical de $U(\mathrm{~g})_{Q}=R$. Alors pour tout idéal premier $P$ de $R / z_{1} R=A$ l'anneau local $A_{\mathbf{P}}$ est régulier.

Glasgow Math. J. 22 (1981) 137-139. 
LEMME. Soit $\mathrm{g}$ une algèbre de Lie nilpotente de dimension finie sur un corps $F$ algébriquement clos de caractéristique 0 . Soit $z$ un élément non nul du centre de $\mathrm{g}$ et soit $P$ un idéal maximal de $U(\mathfrak{g})$ contenant $z-1$. Alors $P$ possède un système privilégié de générateurs $\left(z-1, x_{2}, \ldots, x_{n}\right)$.

Preuve. On procède par récurrence sur la dimension de $\mathfrak{g}$ sur $F$, le cas où $\operatorname{dim}_{F} \mathfrak{g}=1$ étant évident. On peut aussi supposer que $g$ n'est pas abélienne. On considère deux cas; supposons d'abord que $P$ contienne un élément non nul, soit $z_{1}$, du centre de $g$. Puisque $P \neq U(\mathrm{~g})$ on a $1 \notin P$ et donc $z_{1} \neq z$. Notons $\vec{P}$ l'image de $P$ dans $U(\mathfrak{g}) /\left(z_{1}\right)=U\left(\mathrm{~g} / F z_{1}\right)$ : c'est un idéal maximal de $U\left(\mathrm{~g} / \mathrm{Fz}_{1}\right)$ qui contient $\bar{z}-1$ où $\bar{z}$ designe la classe de $z$ modulo l'idéal $F z_{1}$. D'après l'hypothèse de récurrence, $\bar{P}$ possède un système de générateurs privilégiés soit $\left(\bar{z}-1, \bar{x}_{2}, \ldots, \bar{x}_{i}, \bar{x}_{i+1}, \ldots, \bar{x}_{s}\right)$. Alors la suite $z_{1}, z-1, x_{2}, \ldots, x_{s}$ est un système régulier centralisant de générateurs de $P$ et est privilégié.

Montrons que $\left(z-1, z_{1}, x_{2}, \ldots, x_{s}\right)$ est privilégié. Il est certain que si $\mathfrak{Q}$ est un idéal premier de $A=U(\mathrm{~g}) /\left(z-1, z_{1}, \ldots\right)$ alors $A_{\mathfrak{O}}$ est régulier d'après l'hypothèse de récurrence. Si $\mathfrak{Q}$ est un idéal premier de $A=U(\mathfrak{g}) /(z-1)$ alors $A_{\mathfrak{Q}}$ est régulier d'après la proposition 1 et [3].

En second cas supposons que $P$ ne rencontre pas le centre $Z$ de g. Puisque $P$ est un idéal maximal de $U(g), P \cap Z(g)$ est un idéal maximal de $Z(g)$, où $Z(g)$ est le centre de $U(g),[1,4.1 .7]$. Alors, puisque $F$ est algébriquement clos, le centre $Z$ est de dimension 1 sur $F$ et on peut supposer que $Z=F z$.

Il existe dans $\mathrm{g}$ un quadruplet réduisant, $[1,4.7 .7], z, x, y, \mathfrak{h}$ où $[x, y]=z$ où $\mathfrak{h}$ est le centralisateur de $y$ dans $g$ et où $g=F x \oplus \mathfrak{h}$. Si $I$ désigne l'idéal bilatère engendré par $z-1$ dans $U(\mathfrak{g})$ alors l'anneau $U(\mathfrak{g}) / I$ est isomorphe à $A_{1} \otimes_{F} U(\overline{\mathfrak{h}}) /(\bar{z}-1) U(\overline{\mathfrak{h}})$ où $\overline{\mathfrak{h}}=\mathfrak{h} / F y, \bar{z}$ est la classe de $z$ modulo $F y$ et $A_{1}$ désigne l'algèbre de Weyl sur $F$ d'ordre [1, lemme 4.7.8]. Dans l'isomorphisme précédent, l'idéal $P$ a pour image $A_{1} \otimes_{F} \bar{P}^{\prime}$ où $\bar{P}^{\prime}=P^{\prime} /(\bar{z}-1) U(\overline{\mathfrak{h}}), P^{\prime}$ étant un idéal maximal de $U(\overline{\mathfrak{h}})$ contenant $\bar{z}-1$.

D'après l'hypothèse de récurrence, l'idéal $P^{\prime}$ possède un système générateur privilégié commençant par $\bar{z}-1$, soit $\bar{z}-1, x_{2}^{\prime}, \ldots, x_{i}^{\prime}, x_{i+1}^{\prime}, \ldots, x_{i}^{\prime} \in U(\overline{\mathfrak{h}})$. Soit $y_{i} \in U(\mathfrak{g})$ $i=2, \ldots, t$, des éléments tels que $y_{i}=1 \otimes x_{i}^{\prime}$ modulo $(z-1)$.

On vérifie comme en [3] que $\left(z-1, y_{2}, \ldots, y_{t}\right)$ est une suite centralisante. Montrons que cette suite est privilégiée. Si $\mathscr{Q}$ est un idéal premier de $U(\mathfrak{g})$ contenant $(z-1$, $y_{2}, \ldots, y_{i}$ ) alors:

$$
\overline{\mathfrak{Q}}=\frac{\mathfrak{Q}}{(z-1)} \cong A_{1} \otimes \overline{\mathbb{Q}}^{\prime}
$$

où $\overline{\mathcal{Q}^{\prime}}$ est un idéal premier de $\left.U(\overline{\mathfrak{h}}) / \bar{z}-1\right)$. On a aussi

$$
\frac{U(\mathrm{~g})}{\left(z-1, y_{2}, \ldots, y_{i}\right)} \cong A_{1} \otimes \frac{U(\overline{\mathfrak{h}})}{\left(\bar{z}-1, x_{2}^{\prime}, \ldots, x_{i}^{\prime}\right)} .
$$

Posons

$$
A=U(\mathbf{g}) /\left(z-1, y_{2}, \ldots, y_{i}\right) \quad \text { et } \quad B=U(\overline{\mathfrak{h}}) /\left(\bar{z}-1, x_{2}^{\prime}, \ldots, x_{i}^{\prime}\right) .
$$

Notons $\overline{\mathfrak{Q}^{\prime \prime}}$ l'idéal premier de $B$ image de $\overline{\mathfrak{Q}}$ '. 
Par hypothèse de récurrence $B_{\mathbb{S}^{\prime \prime}}$ est régulier. Soit $v_{1}, \ldots, v_{h}$ un système de paramètres de $B_{Q^{\prime}}$ et soit $S=B-{\overline{\mathbb{Q}^{\prime \prime}}}^{\prime \prime}$ alors $S^{-1} A \cong A_{1} \otimes B_{\mathbb{Q}^{\prime}}$. Soit $w_{1}, \ldots, w_{h}, w_{i}=1 \otimes v_{i}$. Alors il est évident que $w_{1}, \ldots, w_{h}$ est un système centralisant régulier de $S^{-1} A$, et donc de $A_{\mathscr{O}}, S^{-1} A$ est un anneau possédant un unique idéal bilatère maximal á savoir $A_{1} \otimes \overline{\Omega^{\prime \prime}}$. $B_{\mathbb{S}^{*}}$ et cet idéal est engendré par $w_{1}, \ldots, w_{h}$. Donc puisque $A_{\mathbb{Q}}$ s'obtient en localisant $S^{-1} A$ en son radical, $\overline{\mathcal{Q}} A_{\mathscr{Q}}$ est engendré par les $w_{1}, \ldots, w_{h}$.

Propostrion 2. Soit g une algèbre de Lie nilpotente sur un corps $F$ algébriquement clos. Tout idéal maximal $P$ de $U(\mathrm{~g})$ possède un système privilégié de générateurs.

Preuve. On raisonne par récurrence sur $\operatorname{dim}_{\mathrm{F}} \mathrm{g}$, la proposition étant évidente si $\operatorname{dim}_{F} g=1$. On suppose $g$ non abélienne. Si $P$ rencontre le centre $Z$ de $g$ en un élément non nul $z$ alors $P /(z)$ est un idéal maximal de $U(\mathfrak{g} / F z)=U(\mathfrak{g}) /(z)$. D'où l'existence d'un système privilégié de générateurs pour $P /(z)$ soit $\left(\bar{z}_{2}, \ldots, \bar{z}_{t}\right)$. Alors $\left(z, z_{2}, \ldots, z_{t}\right)$ est un système privilégié de générateurs pour $P$.

Si $P \cap Z=(0)$, alors la dimension de $Z$ sur $F$ est égal à 1 . Soit $Z=F z$. On peut supposer que $z-1 \in P$, ceci parce que $P$ est maximal, le centre de $U(\mathrm{~g}) / P$ est $F[\mathbf{1}$, Theorème 4.5.7]. Posons $I=(z-1)$; c'est un idéal premier [3] et on applique le lemme.

\section{BIBLIOGRAPHIE}

1. J. Dixmier, Algèbres enveloppantes, (Gauthier-Villars, 1974).

2. T. Levasseur, Séminaire Dubreil $78 / 79$ (Springer-Verlag), à paraître

3. M. P. Malliavin, Régularité locale d'algèbres universelles. C. R. Acad. Sc. Paris t. 283 (1976), 923-925.

4. P. F. Smith, On non commutative regular rings, Glasgow Math. J. 17 (1976), 98-102.

5. R. Walker, Local rings and normalizing sets of elements, Proc. London Math. Soc. (3) 24 (1972), 27-45.

10, Rue Saint Louis en L'Ile

75004 PARIS 